\section{TUMOR CELL-INTRINSIC SIGNALING MEDIATES T CELL EXCLUSION AND PROMOTES RESISTANCE TO CHECKPOINT BLOCKADE THERAPY IN NON-SMALL CELL LUNG CANCER}

Elen Torres-Mejia*, Kim Nguyen, Ellen Duong, Stefani Spranger. MIT, Cambridge, MA, USA

Background Lung cancer is the leading cause of cancer-related death worldwide. ${ }^{1}$ Immunotherapies such as checkpoint blockade therapy (CBT) can be an effective approach to treat patients with metastatic tumors. However, only a fraction of patients is responsive to CBT treatments. ${ }^{2}$ Patients with a non$\mathrm{T}$ cell-infiltrated tumor microenvironment correlate with a poor response to CBT and $\mathrm{T}$ cell infiltration can be influenced by tumor cell-intrinsic signaling pathways. ${ }^{3} 4$ Therefore, understanding the tumor cell-intrinsic mechanisms affecting antitumor immune response will aid us to design better treatments for lung cancer patients. Preliminary work in our group showed that patients with non-small cell lung cancer (NSCLC) can be segregated according to the expression of a $\mathrm{T}$ cell gene signature into $\mathrm{T}$ cell-infiltrated and non- $\mathrm{T}$ cell-infiltrated cohorts. Using an unbiased pathway analysis, we identified that upregulation of specific gene modules significantly correlate with low expression of the $\mathrm{T}$ cell gene signature gene (non-T cell-infiltration). In this project, we aim to investigate how the overexpression of one specific pathway in NSCLC cells impacts the anti-tumor immune responses.

Methods We used a lung cancer cell line derived from a KrasG12D/+ and Tp53-/- mouse (KP) to overexpressed our gene of interest (KP-A). Tumors were induced by subcutaneous or orthotopic implantation and were treated with anti-PD-L1 and anti-CTLA-4 blocking antibodies and analyzed for tumor burden. Infiltration of cytotoxic $\mathrm{T}$ cells and regulatory $\mathrm{T}$ cells were evaluated by fluorescence microscopy. Additionally, we engineered the KP-A and KP cell lines to express the model antigen SIY which allowed us to characterize tumor-specific $\mathrm{T}$ cell responses and utilize SIY-reactive TCR-transgenic T cells.

Results We identified that the overexpression of our gene of interest in KP cells impairs the response to CBT mediated by $\mathrm{T}$ cell exclusion from the tumor. Analyses of tumor-reactive $\mathrm{T}$ cells indicated that $\mathrm{T}$ cell activation and differentiation into effector $\mathrm{T}$ cells was not affected, however, effector $\mathrm{T}$ cells failed to infiltrate KP-A tumors. We are currently investigating the molecular mechanism whereby our gene of interest mediates $\mathrm{T}$ cell exclusion.

Conclusions Our results strongly suggest that tumor cell-intrinsic activation of specific pathways in NSCLC promote immune evasion and contribute to immunotherapy resistance. Understanding the molecular and immunological mechanisms mediating $\mathrm{T}$ cell exclusion from the lung tumor microenvironment will facilitate the development of novel combination treatment strategies for NSCLC patients.

Acknowledgements This work was supported by a postdoctoral fellowship from the Ludwig Center at MIT's Koch Institute for Integrative Cancer Research

\section{REFERENCES}

1.. Chen Z, Fillmore CM, Hammerman PS, Kim CF, Wong KK. Non-small-cell lung cancers: a heterogeneous set of diseases. Nat Rev Cancer 2014;14:535-546.

2.. Hellmann MD, Paz-Ares L, Bernabe Caro R, Zurawski B, Kim SW, Carcereny Costa E, Park K, Alexandru A, Lupinacci L, de la Mora Jimenez E, Sakai H, Albert I, Vergnenegre A, Peters S, Syrigos K, Barlesi F, Reck M, Borghaei $H$, Brahmer JR, O'Byrne KJ, Geese WJ, Bhagavatheeswaran P, Rabindran SK, Kasinathan RS, Nathan FE, Ramalingam SS. Nivolumab plus ipilimumab in advanced non-smallcell lung cancer. N Engl J Med 2019;381:2020-2031.
3.. Chen DS, Mellman I. Elements of cancer immunity and the cancer-immune set point. Nature 2017;541:321-330.

4.. Nguyen KB, Spranger S. Modulation of the immune microenvironment by tumorintrinsic oncogenic signaling. J Cell Biol 2020;219.

Ethics Approval All mouse experiments in this study were approved by MIT's Committee on Animal Care (CAC) DHHS Animal Welfare Assurance \# D16-00078

http://dx.doi.org/10.1136/jitc-2021-SITC2021.320 